\title{
DESPUÉS DE LOS 90: AGENDA DE CUESTIONES EDUCATIVAS
}

GUILLERMINA TIRAMONTI

Flacso - Argentina

tiramonti@flacso.org.ar

El artículo propone una agenda de "cuestiones educativas" orientada a la reconstitución del lazo entre educación y democracia después de las reformas implementadas en los años 90. Construye previamente un estado de situación caracterizado por la presencia de tres procesos: a. un proceso de des-institucionalización escolar que se expresa tanto en la dificultad de la escuela para sostener un marco normativo sancionado socialmente, como en la crisis de identidad por la que atraviesan las instituciones; b. un proceso de fragmentación del sistema educativo que se diferencia de la tradicional segmentación de los sistemas; c. el agotamiento del instrumental teórico-conceptual con el que tradicionalmente nos hemos propuesto organizar y dar sentido a los fenómenos de la realidad. En este marco el artículo propone: I. discutir los limites de la modernidad educativa en países periféricos como el nuestro y, en este marco, los de la pedagogía moderna para superar las problemáticas de discriminación y exclusión educativa; 2. rediscutir el concepto de ciudadanía y el papel de la escuela en la formación ciudadana; 3. repensar y discutir el papel del conocimiento y la investigación en la modificación de las practicas políticas y pedagógicas.

EDUCACIÓN - DEMOCRATIZACIÓN DE LA EDUCACIÓN - SISTEMAS ESCOLARES

\section{ABSTRACT}

AFTER THE 90'S : AN AGENDA ON EDUCATIONAL MATTERS. This article proposes an agenda on "educational matters" which is meant for binding afair education to democracy after the reforms carried out in the 90's. First it describes the current state which is characterized by three processes: a. a school deinstitutionalization process which is expressed both in terms of the difficulty to keep a socially-sanctioned normative framework, and the identity crisis the institutions currently go through; b. a fragmentation process of the educational system which is different from the traditional segmentation of the systems; $c$. the wearing out of the theoretical and conceptual instruments on which we have been traditionally supposed to rely for organizing and making sense out of reality's phenomena. On this framework the article proposes: I. to discuss the limits of educational modernity in peripheral countries such as ours and, in this context, those of modern pedagogy in order to deal successfully with discrimination and educational; 2. to review the concept of citizenship as well as the role ascribed to school in the shaping of citizenship; 3. a second thought and discussion on the role of knowledge and research in changing the practices in politics and pedagogy. EDUCATION - DEMOCRATIZATION OF EDUCATION - SCHOOL SYSTEMS 
En los años 90 los diferentes países de la región implementaron una serie de reformas en sus sistemas educativos nacionales que acompañaron la reestructuración general del modelo de acumulación hasta ese momento hegemónico en la región. Las teorías del capital humano (una versión actualizada con respecto a la de los años 60), los principios de la libre elección y de la racionalidad individual constituyeron los sustentos teóricos de reformas que se proponían aumentar la "competitividad de los mercados" a través de una mejora en la calidad educativa.

Se construyó un paradigma reformista que orientó la agenda de políticas para el sector educativo de los diferentes países de la región. Si bien cada uno de ellos reprocesó las propuestas de acuerdo a su propia matrizsociopolítica, generando diferencias sustanciales en el impacto y en el sentido de muchas de las acciones realizadas, las orientaciones generales fueron compartidas por el conjunto de los países. Los Estados Nacionales se constituyeron en los principales operadores de una agenda de modernización educativa definida en clave organizacional, que se proponía redefinir las funciones del Estado y la política e incluir mecanismos del mercado en la organización de los sistemas educativos.

No es nuestra intención revisar la reforma, sino plantear que estas establecieron no solo una agenda para los Estados sino que además se constituyeron en la referencia casi obligada del discurso de especialistas y académicos. Tanto la discusión política como la académica adquirió en ese periodo o un tono fuertemente ideológico o meramente técnico, generando un ambiente poco propicio para abrirse a nuevos temas de discusión y perspectivas de análisis.

En la Argentina, el agotamiento del impulso reformista y la profundidad de la crisis en que este se da, ha generado un efecto de vaciamiento de la discusión pública sobre temas educativos. Por una parte la reestructuración ha sido tan brutal en nuestro país que es difícil realizar una caracterización del contexto políticoinstitucional en el que los actores se constituyen y actúan y de cuales son las políticas que pueden orientar el campo educativo a favor de la consecución de "objetivos democráticos" si, en definitiva, lo que esta en discusión es justamente la redefinición de la democracia a la luz de los cambios que ha generado en nuestra sociedad el proceso de globalización y la particular forma en que nos incorporamos periféricamente a esta globalización.

¿Desde dónde comenzar a plantear una agenda para la discusión político académica que habilite una reflexión sobre la articulación educación y democracia en el específico contexto de nuestro país? Empezaremos por señalar algunos procesos que arman un estado de situación. 
I. El primer fenómeno a considerar es el de "desinstititucionalización escolar" en el que convergen dos procesos. Uno de ellos es el de la pérdida de eficacia de la escuela para incorporar a las nuevas generaciones al marco normativo imperante, fundamentalmente en el caso de los sectores marginales, pero en general este proceso atraviesa todas las instituciones y esta relacionado con el debilitamiento de la red institucional de la modernidad o la desaparición de sus bases vitales que las transforman según Beck (2000) en "jinetes sin caballos".

En segundo proceso que está en la base del fenómeno de desinstitucionalización y que afecta diferencialmente a el conjunto de las instituciones educativas es la pérdida de especificidad de la función educativa que ha puesto en crisis la identidad social de la escuela. Este fenómeno esta relacionado con la aparición de otros agentes más eficaces en la trasmisión de información y conocimientos, pero también con la multiplicación y heterogeneización de las demandas que procesan las escuelas. Hay en la actualidad una demanda asistencial que pesa sobre las escuelas que se combina y potencializa con un reclamo para que esta compense supuestas deficiencias socializadoras de las familias -, institución que está también en transformación -, reponga los valores que permitan una socialización para la democracia en un marco anómico (?) y de cierto desconcierto valorativo que caracteriza la época. Hay otra serie de elementos que confluyen en la conformación de este fenómeno desinstitucionalizador. La pérdida de perspectiva de futuro para los jóvenes impacta fuertemente en una institución que se justifica por su aporte a la construcción de mejores futuros individuales y sociales. Por otra parte, la ruptura de la articulación positiva entre educación y mejores destinos laborales también aporta a este proceso.

2. El segundo fenómeno que cabe señalar es el de fragmentación de los sistemas escolares. La fragmentación es un fenómeno diferente al de la tradicional segmentación de los sistemas en diferentes circuitos de calidad que se registraron en el país en el momento de la apertura democrática. Los segmentos dan idea de diferencias en el interior de un mismo campo, de la existencia de un todo que de algún modo integra e incorpora, aunque sea diferenciadamente. Lo que estamos presenciando es un proceso distinto donde las instituciones se organizan en referencia directa a sus grupos clientelares (?) construyendo propuestas pedagógicas que no se articulan entre si, con una fuerte impronta reproductora y generando límites muy fuertes a la función de conformación del lazo social que se ha depositado tradicionalmente en la escuela. El quiebre más flagrante en el sistema se da entre instituciones públicas y privadas que está fuertemente referenciado en el origen social de los alumnos. Las escuelas públicas a cargo del Estado están atendiendo cada vez más a 
los sectores más empobrecidos de la población. Frente al discurso que sostiene el contenido ético de un Estado que enfoca su acción en los pobres, es necesario marcar no solo las consecuencias que en materia de integración social resultan de la renuncia de la pretensión de socialización universalista del Estado, sino considerar además el impacto empobrecedor que sobre la prestación educativa genera la falta de pluralidad socio-cultural en las escuelas.

Hay ya suficientes pruebas empíricas en la Argentina para sostener que hemos pasado de un sistema que se proponía la homogenización cultural de la población, a partir de su inclusión en una red más o menos homogénea de instituciones que albergaban una población sociocultural heterogénea en un mismo espacio institucional, a un sistema con diversidad de formatos institucionales pero con homogeneidad sociocultural en el interior de cada institución. La constitución de un heterogéneo mercado educativo que es valorizado desde el paradigma de la libertad de elección en la medida que segrega y discrimina a los grupos sociales para incluirlos en diferentes instituciones anula a la escuela como espacio de integración social.

3. El tercer fenómeno que creo es importante señalar y que emerge de los 90 es el agotamiento de nuestros marcos conceptuales. El instrumental teóricoconceptual y las metodologías de análisis que utilizamos para ir al campo se muestran insuficientes o no aptos para dar cuenta de la complejidad de fenómenos y situaciones que se dan en la realidad y poco potentes para construir sentidos en base a estos fenómenos y situaciones. En esta dificultad confluyen muchos elementos: a. la reestructuración del orden global que transforma los marcos que regulan la acción colectiva y redefine las relaciones sociales; b. la especificidad del impacto de la globalización en nuestra región que requiere por lo menos una redefinición de los marcos conceptuales provenientes de los países productores de teoría que son afectados de un modo muy distinto por este fenómeno. Si bien esta crisis de los marcos conceptuales no es privativa de nuestra región, los países centrales están generando una rica bibliografía que intenta interpretar y construir sentidos alrededor del nuevo orden mundial y de los fenómenos puntuales que afectan la vida cotidiana. Esta producción no siempre es pertinente para nuestra realidad; c. la insuficiencia de la investigación cientíica en la región resultante, de la escasa inversión en ciencia y técnica que en general hacen los Estados y del desplazamiento de las financiaciones de las fundaciones de la investigación a proyectos de acción.

En base a estos procesos, proponemos una agenda de cuestiones a discutir en el campo educativo. No se trata de una agenda de políticas para el sector sino de un programa de investigación y reflexión que nos permita renovar las articulaciones 
hasta ahora pensadas entre educación y democratización social. Lo que hago aquí es señalar algunos temas para la discusión.

I. Discutir los límites de la modernidad educativa en países periféricos como el nuestro y en este marco los de la pedagogía moderna para superar las problemáticas de discriminación, exclusión, segmentación y fragmentación educativa. En la década del 80 los países de la región seguían registrando altas tasas de repetición y deserción y los sistemas educativos estaban claramente segmentados en razón del origen social de los alumnos. Estos datos no fueron utilizados para discutir el modelo pedagógico que generaba tales fenómenos sino que fueron interpretados como indicadores de la "deficiencia" en la aplicación del mismo y desde esta postura se diseñaron las reformas de los 90. América Latina, y en especial Brasil, tiene antecedentes en la búsqueda de pedagogías alternativas que permitan superar las tendencias discriminadoras del sistema. En esta línea de trabajo habría que insistir por supuesto a la luz de la globalización, los cambios en la circulación del conocimiento y la información, las nuevas dimensiones de la alfabetización, etc.

Paradójicamente las reformas si discutieron el andamiaje institucional generado por la modernidad para el desarrollo de los sistemas educativos. Estamos haciendo referencia al papel que jugaron los Estados estados nacionales en la producción de un campo de integración y sentido en el que se desenvolvió la acción educativa. Las situaciones nacionales son muy diferentes entre sí. La tendencia generalizada en la región fue a la conformación de sociedades duales donde una parte de la población era articulada a la red del Estado y el mercado formal y definía su identidad en relación a esta doble referencia y el resto se articulaba a una heterogénea red de organizaciones de la sociedad civil. Por supuesto no se trata de redes claramente diferenciadas y sin relación mutua sino que se conforman en diálogo una con otras. Lo que importa es señalar que las condiciones en que se constituye la ciudadanía en uno y otro espacio son diferentes, así como lo son los procesos de individualización a los que son sometidos los que habitan en uno u otro espacio. Si bien para ambos se trata de un proceso de auto confrontación en el que los individuos están condenados a ser ellos mismos (Senentt, 1978) sin embargo en un caso este se desenvuelve en un marco de socialización asistida y en otro desreglado lo que aumenta significativamente las inseguridades antológicas (Robles, 1999).

La reestructuración de fin de siglo $X X$ no modificó esta situación de dualización de la sociedad, por el contrario la profundizó - en la medida que la brecha de desigualdad se hizo mayor - y afectó a países que antes no estaban en esa situación, y por otro lado cambió la "matriz sociopolítica" en la que la dualización se desenvuelve. 
Se pasó de una sociedad estadocéntrica o político céntrica (Garretón, 1998) a una sociedad caracterizada por la "diseminación" de espacios y "formatos de intercambio político" (Arditi, 1997). Este cambio de (?) matriz sociopolítica se realiza además en el marco de la globalización que ha generado condiciones más favorables para la expansión del mercado y sus lógicas y estrechado los márgenes de autonomía de la política para definir líneas de acción desde el Estado.

Las reformas introdujeron en el sistema escolar lógicas de mercado que los reconfiguraron trasladando, en gran medida, a las organizaciones de la sociedad civil y los individuos el control del sistema. En algunos casos esto acompaña o tiene un sentido de democratización del espacio de toma de decisiones en otros se trata simplemente de devolución a la sociedad de funciones que antes eran atendidas por el Estado.

En la matriz sociopolítica anterior, la educación pública fue un arma de centralización política y un dispositivo de disciplinamiento (?) disciplina social pero también de promoción democrática de la sociedad (De Certeau, 1984). La pregunta de ahora en más es iqué papel juega la escuela en los procesos por un lado de reproducción social y por otro de disciplinamiento (Íd.) de la sociedad? Con esto queremos señalar que desaparecido o debilitado el vínculo Estado-Escuela y dada la centralidad que hasta ahora ha tenido esta ligazón para el gobierno de una sociedad de hombres libres, cabe preguntarse si la escuela cumple hoy alguna función en la construcción de la gobernabilidad y en la estructuración de una sociedad y de ser así icual es su específica contribución en este aspecto?

Por otro lado icuáles son las alternativas pedagógicas que permitan procesar de un modo más igualitario las profundas desigualdades que están presentes en nuestras sociedades? ¿Cuál es la posibilidad democratizadora de la escuela en términos de acceso al conocimiento socialmente reconocido como válido y de desenvolvimiento, actualización y enriquecimiento del propio capital cultural?

2. Rediscutir el lugar de la escuela en la conformación de iqué ciudadanía? Si hay una categoría que ha estallado con la globalización es la de "ciudadanía". concepto de ciudadanía esta ligado a la noción de vínculo con la comunidad y a la idea de derechos individuales (Kymlicka, Norman, 1997) y a la secularización de la esfera pública. Cada una de estas dimensiones de la ciudadanía tuvo como referente al Estado y este, a su vez, hizo de los sistemas educativos un dispositivo de concreción.

La primera de estas dos dimensiones esta relacionada con la constitución del sentido de pertenencia a colectivo con el que se comparten determinados rasgos identitarios (?) que difieren de los de otros grupos. La constitución de la ciudadanía 
estuvo íntimamente relacionada con la conformación de la nacionalidad y esta con la identificación de un cuerpo de valores, principios, creencias, representaciones históricas, tradiciones, representaciones del ser nacional con las que se identifican los diferentes miembros de una sociedad. El sentido de pertenencia es un elemento decisivo en la construcción del lazo social. La escuela jugó un papel central en el desarrollo de esta dimensión de la ciudadanía. La escuela fue vehiculadora de esta pertenencia o esta creencia, los rituales, la invención de las tradiciones, de un pasado que nos es común, la identificación de los valores que nos distinguen y de los rasgos que nos son propios tuvieron como finalidad generar un sustrato cultural en el que nos podíamos reconocer y al que podíamos identificar como propio. Por supuesto se trata de un arbitrario cultural que expresa de un modo desigual los valores e intereses de los diferentes sectores de la sociedad, pero se presenta como un universal. Esta función nacionalizadora de la escuela esta doblemente discutida.

Primero se discute la capacidad de la escuela de intervenir en la conformación de las subjetividades y por tanto de imponer este arbitrario cultural. Los medios de comunicación masiva estarían cumpliendo esta función de incorporación de los individuos a un determinado arbitrario cultural. En segundo lugar se discuten los espacios para la constitución de la pertenencia. La disyuntiva de "patriotas o cosmopolitas" da cuenta de uno de los clivajes que la globalización genera en la ciudadanía, pero no la única ya que los comunitaristas han planteado la pertenencia con alcances circunscriptos a las sociedades locales donde la relación es aún cara a cara. La globalización a reposicionado lo local y con ello discute los alcances de la pertenencia.

Las políticas de descentralización y autonomía escolar que plantean una rearticulación de la escuela con el espacio comunitario redefinen la referencia espacial de la acción socializadora de la escuela, a la vez que propician una alianza escuela comunidad que remplaza la anterior escuela estado nacional generando un nuevo ámbito de control y de resolución de la pertenencia.

Como se articulan escuela y medios masivos de comunicación? i es posible pensar en una relación dialógica entre escuela y medios masivos donde la primera aporte a la construcción de un interlocutor capaz de interpretar la información y establecer una distancia crítica con respecto a ella y participar más activamente en la conformación de la pertenencia? Esto abre la discusión sobre política y mass media.

A su vez es lo nacional el espacio de construcción de la pertenencia? $\bigcirc$ es posible pensar en identidades múltiples que incluyan a su vez pertenencias, locales, nacionales y trasnacionales. Cuales serían entonces el conjunto de valores y creencias alrededor de los cuales se teje la pertenencia? En las sociedades fragmentadas como 
las nuestras iexisten espacios de construcción de la pertenencia que sobrepasen la frontera del fragmento y hay alguna oportunidad para la escuela de construir puentes entre ellos o como dice Duschatzky ( 1999) la escuela es la frontera?

La ciudadanía moderna esta ligada a la conformación de un sujeto de derecho y este a la existencia de un estado garante del derecho. En la opinión de Marshall, la ciudadanía consiste esencialmente en asegurar que cada cual sea tratado como miembro pleno de una sociedad de iguales. La ciudadanía supone otorgar a los individuos una suma de derechos que el autor dividió en tres categorías -, civiles, políticos y sociales -, que exigen la contraparte del Estado como garante del ejercicio de los derechos. La constitución de "sujetos de derecho" supone una relación o articulación de los individuos con el poder mediado por la ley y no por las relaciones tutelares. Es la norma la que establece los derechos a los que los individuos pueden acogerse por su condición ciudadana independientemente de su condición socio-económica.

La educación no solo formó parte del paquete de derechos que debían ser garantizados al conjunto de los ciudadanos sino que además generó en la población continuidades culturales que se constituyeron en el soporte de la pretensión de la igualdad ciudadana. Esta dimensión de la ciudadanía funciona en la medida que existe un Estado garante y políticas universalistas que a la vez que posibilitan una socialización común articulan al conjunto de los sectores de la población al ejercicio de derechos que también les son comunes. En América Latina, donde imperan las sociedades dualizadas (?) esta fue una pretensión con poco nivel de realización. De modo que coexistieron ciudadanías diferenciadas y una escasa autonomía del status ciudadano del económico.

La articulación de las sociedades periféricas al mundo globalizado generó mayor concentración de ingresos profundizando las diferencias ya existentes. En el mismo proceso y en el marco de la reconfiguración del Estado Nacional y de la distribución de espacios con el mercado, la acción educativa "publica" se enfocó en la asistencia educativa a los pobres a la vez que se proponía una oferta privada para la población con capacidad de financiamiento de su educación. El estado renuncia así a una socialización abarcadora del conjunto de la población y condiciona la dualización de la ciudadanía que se fragmenta entre los asistidos y los consumidores. Se rompe la posibilidad de la socialización conjunta y sobre todo del "derecho igualitario" como elemento que define la ciudadanía.

Aquí nuevamente la pregunta está centrada en como se articula esta ciudadanía con la democracia. Desde la "ciudadanía" como consumo, la democracia puede ser pensada como sinónimo de acceso a un gran número de productos entre los cuales 
está la educación. La democracia se desvincula de cualquier ideal de justicia, igualdad y derechos sociales (Ortiz, 1996) y se transforma en esta posibilidad de elección. Las propuestas de organización del sistema en base a la libre elección se apoyan en esta concepción de la ciudadanía.

¿Se puede asimilar consumo a ciudadanía? ¿los requerimientos para su ejercicio son los mismos? ilibertad de elección, entendida como selección de productos, es asimilable a un ejercicio de la libertad que reconoce en la igualdad su punto de tensión y límite? Es posible pensar en una socialización para la democracia, en el contexto de sociedades fragmentadas, donde no exista un principio compartido de justicia al cual apelar para establecer condiciones que hagan posible el intercambio entre el conjunto de los ciudadanos? itiene la escuela algún lugar en la reposición de este principio y por tanto es posible pensar en una acción de la escuela la cuál se contraponga a las tendencias de dualización de la sociedad?

Si suponemos que la educación es un campo específico donde se procesan de un modo particular las tendencias que atraviesan al conjunto del sistema, podemos construir una hipótesis de márgenes de autonomía realizables a través de específicas propuestas pedagógicas. Sin embargo, es necesario considerar que la sociedad remodela los valores y los redefine, adecuándolos a las necesidades y los intereses de las fuerzas sociales en conflicto. De modo que las democracias se han redefinido a la luz de una nueva articulación con el mercado que las transforma en un atributo del mismo, que estrecha los márgenes de autonomía y que exige una redefinición de la ciudadanía que considere este nuevo status del mercado.

Repensar la ciudadanía democrática y el lugar de la escuela en la formación de esta ciudadanía remite a la discusión sobre los valores alrededor de los cuales esta se constituye y de su expresión en los principios que organizan el sistema educativo. En ambos casos, en los años 90, asistimos a una redefinición de los sentidos y alcances de los valores que se sustentaban desde los discursos políticoeducativos, hubo desplazamientos y nuevas presencias.

Un ejemplo de esta redefinición valorativa es la presencia que los conceptos de "competitividad" y "equidad" adquirieron en el discurso educativo.

La "competitividad" incluye una dimensión en la socialización ciudadana que rescata a la competencia como medida del valor de la educación en general y de las capacidades individuales en particular. La competencia deja de ser un criterio distributivo propio del mercado para transformarse en un valor que legitima la distribución del conjunto de los bienes sociales. Una sociedad "ordenada por la competencia" es diferente a aquella donde prima el mérito. El mérito puede estar 
definido en cada esfera de la sociedad de acuerdo a principios que le son propios. Una sociedad competitiva en cambio no reconoce la existencia de esferas diferentes con capacidad de definir sus propios criterios de mérito sino que adopta el principio del mercado como medida de las realizaciones en cualquiera de las esferas del hacer de la sociedad y también como criterio para la definición de la ciudadanía.

Del mismo modo una sociedad equitativa es diferente de una sociedad de iguales. El principio de "igualdad" establece límites a las distancias sociales, marca fronteras a las desigualdades de hecho y las transforma en patologías que exigen remedio?. La "equidad" no tiene esta connotación, no genera ningún compromiso por los resultados solo propone equivalencia en los recursos que se ponen a disposición de los individuos (Garretón, 1998). La "equidad" no establece límites a las situaciones de desigualdad, solo exige una compensación de los recursos disponibles para la socialización competitiva. La equidad permite reconocer la diversidad de intereses, de referencias identitarias (?) y también de las desiguales distribución de bienes y reconocimiento social.

Hasta aquí, hemos planteado la cuestión de los valores y como estos se asocian a una redefinición de la democracia que legitima otro lugar para el mercado. En este caso, el punto de discusión es la condición democrática de una sociedad organizada alrededor de estos valores y la contribución de la escuela en esta construcción.

En los últimos años se ha generado una nueva cuestión alrededor de los valores y la educación más asociada a los problemas de la gobernabilidad a secas que al sentido democrático de la socialización escolar. La discusión refiere a las tendencias de desintegración y ruptura del lazo social que ha generado en las sociedades periféricas su incorporación al proceso de mundialización globalización y a la necesidad de transformar a la escuela en un espacio donde se recree la virtud ciudadana de la "solidaridad" y un núcleo de valores morales a partir de los cuales regenerar el entramado social. El ejercicio de la solidaridad es pensado como constructor de un lazo que relaciona a los extremos de la escala social permitiendo que estos se reconozcan como miembros de un mismo espacio social. Desde estas posiciones la solidaridad no esta reñida con la aceptación de lo desigual. No pone en cuestión la situación desigual sino que se propone construir una relación allí donde no la hay e intenta contrarrestar los riesgos de las rupturas del entramado social. La pregunta es en este caso si la solidaridad es una forma relacional compatible con la socialización democrática?

En el reclamo por la recuperación de un espacio de evangelización en un núcleo de principios morales destinados reponer en las conductas la orientación de 
los valores, se actualizan fantasías autoritarias, reacciones a la manifestaciones de violencia que genera la ruptura del lazo social y la demanda por una restitución de un espacio de control social. La propuesta puede ser discutida con diferentes argumentos. El primero es el de la dificultad de pensar un espacio de reposición de valores con autonomía de aquellos que han redefinido el sentido de la democracia y que se sostiene desde la sociedad mediática. ¿Puede pensarse en una escuela de la contracultura? iEs posible un espacio de la contracultura cerrado a la influencia del conjunto de valores en que se sustenta la práctica social? Es verdad que la propuesta da cuenta de un proceso de desinstitucionalización que habla de la disolución de los marcos institucionales de referencia para orientar las conductas, pero de ser así, el lugar de la escuela es el de reposicionamiento de los valores del orden o de reflexión sobre los valores en los que se sostiene el orden. iSe trata de un reclamo por la reposición del orden o por una construcción diferente que exige un cuestionamiento de los soportes valorativos del orden?

Finalmente esta discusión sobre los sentidos de la ciudadanía no solo compromete una revisión de la articulación ciudadanía y mercado, ciudadanía y asistencia sino que además exige una reflexión crítica sobre los límites de una ciudadanía moderna en las sociedades periféricas. Retomamos la idea de límites que rescata los principios de la modernidad como criterio organizador de la sociedad.

4. Repensar y discutir el papel del conocimiento y la investigación en la modificación de las prácticas políticas y pedagógicas. El desarrollo de la investigación social en América Latina se legitimó en base a su capacidad de producir un conocimiento que permitiera intervenir para modificar los comportamientos sociales y de este modo posibilitar la construcción de sociedades modernas. La investigación social ató de este modo su legitimidad a su capacidad de generar cambios en la sociedad. En el campo educativo la investigación que se desarrolla a partir de los años 60 esta fuertemente articulada y justificada por la posibilidad de orientar reformas educativas que modernizaran el sistema. De modo que la investigación estuvo tensa desde un comienzo por la doble demanda de la intervención en las prácticas y la orientación modernizadora de esa intervención.

Por otra parte la imbricación del discurso pedagógico con el pastoral le otorgó a la investigación de este campo una orientación a la acción reformista que permeó de prescripción buena parte de su discurso.

En los años 90 se desarrolló en la región un discurso que cuestionó el valor de la investigación educativa y se construyó un discurso de deslegitimación de esta práctica dada su escasa relevancia y su escasa contribución al mejoramiento de las 
políticas y de las prácticas pedagógicas (Palamidessi, 2002). En este año Guillermo Briones ( 1990) publica una investigación de profusa difusión en la región que mostraba la escasa o nula utilización de la investigación por parte de los tomadores de decisiones. Hay numerosos trabajos de Brunner (2000) que abonan esta tesis de "falta de utilidad" o "irrelevancia" de la investigación educativa. Estas tesis a la vez que ilegitimaban la producción académica habilitaban la reconversión de los perfiles académicos a la de "técnico político" entendiendo por este un investigador portador del conocimiento dedicado a actuar tanto en la gestión gubernamental como en el asesoramiento a organismos internacionales.

En los mismos años, se implementó en la región una reforma educativa que como las que la precedieron se justificaba por su potencialidad modernizadora, en este caso, se trataba de una modernización de la gestión de los sistemas que acompañaba la articulación de la región al mundo globalizado.

El discurso reformista es alimentado por una serie de papers generados en el seno de los organismos internacionales en los que se marcan las exigencias y desafíos para América Latina en el campo educativo y se marcan las orientaciones de políticas para la región. Junto con ello hay una multiplicación de la producción de eventos internacionales en la que se construye "un sentido común" (Tiramonti, 200 I) reformista construido sobre la base de estas prescripciones y con escasa intervención de la investigación académica.

La producción de los organismos internacionales fue acompañada por otra serie de documentos e investigaciones empíricas que se desarrollaron en el seno de una nueva red de las $\mathrm{ONG}$ del conocimiento encargadas de producir una investigación empírica y discurso técnico legitimador de las propuestas promovidas por los organismos internacionales en la región.

El desarrollo de esta nueva red de think tanks ha generado un mercado diversificado para la producción de conocimiento educativo en el que coexisten estos nuevos espacios, con la producción en el seno de las universidades, anteriores centros de investigación y formación y la producción de los organismos oficiales'.

La pregunta es en qué espacios y con qué financiamiento abordar un desarrollo de la investigación educativa que permita construir un conocimiento que de cuenta

I A diferencia de lo que pasó en Chile donde el Estado generó una demanda importante de investigación en las ONG en la Argentina el Estado se constituyó en el empleador de buena parte de los profesionales de este campo. En ambos casos se generó una "cuestión" alrededor de la autonomía del campo de la investigación educativa (ver Palamidessi, 2002). 
de los fenómenos que hoy atraviesan nuestros sistemas educativos. Cuáles son las políticas que pueden generar condiciones favorables para esta producción?

Por otra parte habría que rediscutir los fundamentos legitimadores de la producción de investigación educativa a la luz de los límites de las pretensiones modernizadoras que le proporcionaron sentido en etapas anteriores.

\section{BIBLIOGRAFÍA}

ARDIT, B. La Mutación de la política: un mapa del escenario post-liberal de la política. Revista Nueva Sociedad, Caracas, n. I 50, p. I0-23, 1997.

BECK, U. La Democracia y sus enemigos. Barcelona: Paidós, 2000.

BRIONES, G. Generación, diseminación y utilización del conocimiento en educación. Santiago de Chile: Flacso, 1990.

BRUNNER, J. J. Nuevas tecnologías y sociedad de la información. Santiago de Chile, 2000. (Documento Preal, n. 16).

DE CERTEAU, M. La Cultura en plural. Buenos Aires: Nueva Visión, 1984.

DUSCHATZKY, S. La Escuela como frontera. Buenos Aires: Paidós, 1999.

GARRETÓN, M. A. (coord.). América Latina: un espacio cultural en el mundo globalizado. Colombia: Convenio Andrés Bello, 1998.

KYMLICKA, W.; NORMAN, W. El Retorno del ciudadano: una revisión de la producción reciente en teoría de la ciudadanía. Buenos Aires: Ágora, 1997.

ORTIZ, R. Otro território: ensayos sobre el mundo comtemporáneo. Buenos Aires: Universidad de Quilmes, 1996.

PALAMIDESSI, M. La Investigación educacional en la Argentina. Argentina: Flacso, 2002.

ROBLES, F. Los Sujetos y la cotideaneidad: elementos para unna microsociología de lo comtemporáneo. Santiago de Chile: CEl; Sociedade Hoy; Universidad de Concepción, 1999.

SENNETT, R. El Declive del hombre público. Barcelona: Península, 1978.

TIRAMONTI, G. El Papel del Banco Mundial en la construcción de la agenda educativa de América Latina. Cuardernos de Pedagogía, Madrid, 2001.

Recebido em: abril 2003

Aprovado para publicação em: abril 2003 